\title{
Assessment of rheological behavior of secondary polymeric raw materials in the conditions corresponding to processing of polymers by method of extrusion and injection molding
}

\author{
R. Y. Lazdin, V.P. Zakharov, A. S. Shurshina, E. I. Kulish ${ }^{\dagger}$ \\ †onlyalena@mail.ru
}

Bashkir State University, 32 Zaki Validi St., Ufa, 450076, Russia

\begin{abstract}
In the present work, rheological behavior of polymeric raw materials in the conditions corresponding to processing of polymers by the methods of extrusion and injection molding is modeled. Mixes based on secondary polypropylene and the following polymers: secondary polyethylene of low pressure LDPE-276 and secondary polyethylene of high pressure HDPE-20 were used as a polymeric basis. The composites were obtained in a melt using the laboratory station "PlastographEC" (Brabender, Germany) in $15 \mathrm{~min}$ at a load of $200 \mathrm{~N}$, temperature of $180^{\circ} \mathrm{C}$ and screw speed of 30 revolutions per minute. Rheological measurements were taken in the oscillation mode on a modular dynamic rheometer Haake MarsIII at $220^{\circ} \mathrm{C}$ in a oscillation frequency range from 0.01 to $100 \mathrm{~Hz}$. It is revealed that with an increase in content of the second polymer in the mix an increase in the values of complex viscosity of the melt always takes place. This is apparently due to the fact that the samples of the second polymers in all cases were characterized by a higher value of the melt viscosity, than the secondary polypropylene. In addition,an increase in the content of the second polymer in the mix led to an increase in the values of exponent $n$ in the frequency dependence of the viscosity that confirms the strengthening of the dependence of viscosity on the frequency of oscillations and a deviation of melt viscosity of composition from the behaviour typical of a Newtonian liquid. It is shown that compositions based on mixes of polypropylene with HDPE-20 and LDPE-276 can be fairly easily processed at high shear rates, while the enhanced interaction of polymers will provide them improved deformation and strength characteristics.
\end{abstract}

Keywords: mix of polymers, secondary polymers, rheology.

УДК: 541.64:536

\section{Оценка реологического поведения вторичного полимерного сырья в условиях, соответствующих переработке полимеров методом экструзии и литья под давлением}

\author{
Лаздин Р. Ю., Захаров В. П., Шуршина А. С., Кулиш Е. И. ${ }^{\dagger}$ \\ †onlyalena@mail.ru
}

Башкирский государственный университет, ул. Заки Валиди, 32, Уфа, 450076, Россия

\begin{abstract}
В работе осуществлено моделирование реологического поведения полимерного сырья в условиях, соответствующих переработке полимеров методами экструзии и литья под давлением. В качестве полимерной основы были использованы смеси на основе вторичного полипропилена и следующих полимеров: вторичного полиэтилена низкого давления ПНД-276 и вторичного полиэтилена высокого давления ПВД-20. Получение композиционных материалов осуществляли в расплаве на лабораторной станции «PlastographEC» (Brabender, Германия) в течение 15 минут при нагрузке $200 \mathrm{H}$ при температуре $180^{\circ} \mathrm{C}$ и скорости вращения шнеков 30 оборотов в минуту. Реологические измерения проводили в осцилляционном режиме на модульном динамическом реометре Haake MarsIII при $220^{\circ} \mathrm{C}$ в диапазоне частот осцилляции от 0.01 до 100 Гц. Обнаружено, что по мере увеличения содержания второго полимера в смеси всякий раз имело место увеличение значений комплексной вязкости расплава, очевидно, вследствие того, что об-
\end{abstract}


разцы вторых полимеров во всех случаях характеризовались большими значениями вязкости расплава, нежели вторичный полипропилен. Кроме того, увеличение содержания второго полимера в смеси приводило к увеличению значений показателя $n$ в частотной зависимости вязкости, свидетельствующего об усилении зависимости вязкости от частоты осцилляции и отклонении вязкости расплава композиции от поведения, характерного для ньютоновской жидкости. Показано, что композиции на основе смесей полипропилена с ПВД-20 и ПНД-276 будут достаточно легко перерабатываться при высоких скоростях сдвига, а повышенное взаимодействие полимеров между собой обеспечит им улучшенные деформационно-прочностные характеристики.

Ключевые слова: смеси полимеров, вторичные полимеры, реология.

\section{1. Введение}

Известно, что при производстве изделий из полимеров экструзионным методом диапазон реализуемых скоростей сдвига варьируются от 0.01 до $1000 \mathrm{c}^{-1}-$ он различен при течении расплава вдоль канала шнека, при перемешивающем течении, перпендикулярном оси канала, и при обратном потоке, от любого витка шнека к ведущим канавкам [1-3]. В процессе производства полимерных изделий методом литья под давлением также не существует какой-либо единственной скорости сдвига, а имеет место диапазон скоростей сдвига: впрыскивание через литьевое отверстие капиллярного типа - до $10000 \mathrm{c}^{-1}$, течение при заполнение литьевой формы - примерно $100 \mathrm{c}^{-1}$, течение после снятия давления (для обеспечения полного заполнения литьевой формы и предотвращения температурной усадки горячего расплава в холодной форме) - менее $1 \mathrm{c}^{-1}$.

Фактически, для полного суждения о реологических свойствах полимеров надо иметь кривую течения при изменении скорости сдвига в диапазоне 3-4 десятичных порядков. Ни один из существующих приборов не в состоянии надежно провести измерения в таком широком диапазоне скоростей сдвига. В связи с этим, возникает необходимость моделирования реологического поведения полимерного сырья в условиях, соответствующих переработке полимеров методами экструзии и литья под давлением. Учитывая, что лидирующее место среди промышленно выпускаемых крупнотоннажных полимеров занимают полиолефины [4-7], что обусловлено их низкой себестоимостью и химической инертностью $[5,8]$, а модификация полиолефинов путем их физического смешивания представляется наиболее простым и низко затратным способом создания новых материалов [9-19], в качестве полимерной основы для проведения подобного рода исследования были использованы смеси наиболее распространенного крупнотоннажного полиолефина - полипропилена (ПП), который использовался в виде вторичного полимерного сырья и следующих полимеров: вторичного полиэтилена низкого давления ПНД-276 и вторичного полиэтилена высокого давления ПВД-20.

Использование вторичных полимеров особенно актуально с точки зрения их утилизации, поскольку традиционными методами полимерные отходы сложно утилизировать. Для вторичного использования утилизированных пластмасс достаточно стимулов: экологический аспект, спрос потребителей, требования законодательства и низкая стоимость. Положительной стороной использования вторичных полимеров является также и то, что получается дополнительное количество полезных продуктов для различных отраслей народного хозяйства и не происходит повторного загрязнения окружающей среды. Кроме того, вторично переработанные пластмассы можно использовать в большинстве технологических процессов, включая литье под давлением, компрессионное прессование, экструзию, каландрование и т. д. Основная проблема, которая может возникнуть при использовании вторичного полимерного сырья, это сложность их переработки в связи с неопределенными реологическими характеристиками вторичных полимеров.

В связи с этим целью работы является исследование реологического поведения вторичных полимеров (полипропилена и полиэтилена) в условиях, соответствующих переработке полимеров методом экструзии и литья под давлением

\section{2. Экспериментальная часть}

В работе использовали образцы вторичных полимеров: ПП, соответствующий первичному ПП марки FF/3350, образец вторичного ПНД-276, соответствующий первичному ПЭ марки ПЭНД 273-83, образец вторичного ПВД-20, соответствующий первичному ПЭ марки ПВД 10803-020, представляющие собой дробленый материал из некондиционных изделий, производимых методом литья под давлением в технологическом производстве ООО «ЗПИ Альтернатива» (Россия, Республика Башкортостан, г. Октябрьский).

Получение композиционных материалов осуществляли в расплаве на лабораторной станции «PlastographEC» (Brabender, Германия) в течение 15 мин при нагрузке $200 \mathrm{H}$ при температуре $180^{\circ} \mathrm{C}$ и скорости вращения шнеков 30 оборотов в минуту. Количество загружаемого полимера составляло 25 г.

Реологические измерения проводили в осцилляционном режиме на модульном динамическом реометре Haake MarsIII при $220^{\circ} \mathrm{C}$ в диапазоне частот осцилляции от 0.01 до 100 Гц.

Представляя полученные экспериментально значения вязкости от частоты осцилляции $\eta \sim f^{n}$ в логарифмических координатах $\lg \eta=n \lg f+$ const можно получить линейное уравнение, позволяющее рассчитать значение $n$, как тангенс угла наклона и значение вязкости при высоких значениях частот осцилляции (например, 1000 Гц), которое нельзя определить экспериментально. Такие расчетные значение вязкости $\eta^{\text {расч }}$ были определены для расплавов ПП, ПВД-20 и ПНД-276, а также их смесей. Значение вязкости $\eta^{\text {ад }}$ рассчитывали как аддитив- 
ное, исходя из соотношения компонентов смеси и рассчитанных значений для индивидуальных полимеров. Например, для смеси состава $80 \%$ ПП - 20\% ПВД-20 значение $\eta^{\text {ад }}=0.8 \times \eta_{\Pi \Pi}^{\text {pacч }}+0.2 \eta_{П в Д-20}^{\text {pacч }}$.

\section{3. Обсуждение результатов}

Осуществить принципиальную возможность моделирования поведения расплавов полимеров в той области скоростей сдвига (или частоты осцилляции, а стало быть, и угловой скорости), при которых проводится переработка полимеров методами экструзии и литья под давлением, возможно установив закономерность изменения вязкости с изменением скорости сдвига.

Известно, что только в том случае, когда жидкость является чисто вязкой, ньютоновской, вязкость является величиной постоянной, независящей ни от скорости сдвига, ни от частоты осцилляции. При этом, если представить вязкость как функцию частоты (угловой скорости) $\eta \sim f^{n}$, где $f$ - частота осцилляции, то показатель $n=0$. Если же жидкость не является ньютоновской, т.е. представляет собой вязкоупругую жидкость (а, как известно, расплавы полимеров представляют собой именно вязкоупругие жидкости), вязкость зависит от частоты осцилляции (угловой скорости). При этом, $\eta \sim f^{n}$ и $n \neq 0$, а для полимеров, которые, как правило, представляют собой псевдопластичные жидкости, $n<0$. Таким образом, чем больше отклоняется значение $n$ от нуля, тем сильнее выражена аномалия вязкости.

Для определения показателя $n$ в частотной зависимости вязкости были сняты зависимости комплексной вязкости от частоты осцилляции для анализируемых нами композитов ПП-ПНД-20 и ПП-ПНД-276, полученные данные были обработаны в логарифмических координатах, что позволило определить параметр $n$ как тангенс угла наклона (Табл. 1).

Как показали проведенные исследования, для всех изученных систем наблюдался ряд общих закономерностей. Во-первых, по мере увеличения содержания второго полимера в смеси всякий раз имело место увеличение значений комплексной вязкости расплава, очевидно, вследствие того, что образцы вторых полимеров во всех случаях характеризовались большими значениями вязкости расплава, нежели вторичный ПП. Во-вторых, по мере увеличения содержания второго полимера в смеси имело место увеличение значений показателя $n$ в частотной зависимости вязкости, свидетельствующее об усилении зависимости вязкости от частоты осцилляции и отклонении вязкости расплава композиции от поведения, характерного для ньютоновской жидкости,

табл. 1. Значения степенного показателя $n$ в частотной зависимости комплексной вязкости, определенной из реологических данных, проведенных в осцилляционном режиме, для композиции на основе смесей полимеров.

Table 1. Values of a degree index $n$ in the frequency dependence of the complex viscosity defined from the rheological data which are carried out in the oscillation mode for composition based on the mix of polymers.

\begin{tabular}{|c|c|c|c|c|}
\hline $\begin{array}{l}\text { Второй полимер } \\
\text { Second polymer }\end{array}$ & $\begin{array}{c}\text { Содержание второго поли- } \\
\text { мера в смеси, \% мас. } \\
\text { Content of the second } \\
\text { polymer in the mix, wt.\%. }\end{array}$ & $n$ & $\begin{array}{c}\eta^{\text {pacu }},(\Pi a \cdot c) \\
\eta^{\text {cal }},(\mathrm{Pa} \cdot \mathrm{s})\end{array}$ & $\begin{array}{l}\eta^{\text {ад }},(\Pi a \cdot c) \\
\eta^{\text {ad }},(\mathrm{Pa} \cdot s)\end{array}$ \\
\hline \multirow{6}{*}{$\begin{array}{l}\text { ПВД-20 } \\
\text { HDPE-20 }\end{array}$} & 5 & 0.15 & 65.45 & 66.29 \\
\hline & 10 & 0.16 & 65.80 & 66.54 \\
\hline & 20 & 0.19 & 66.32 & 67.00 \\
\hline & 40 & 0.29 & 66.88 & 67.95 \\
\hline & 10 & 0.38 & 67.98 & 70.32 \\
\hline & 100 & 0.43 & 70.79 & 70.79 \\
\hline \multirow{6}{*}{$\begin{array}{l}\text { ПНД-276 } \\
\text { LDPE-276 }\end{array}$} & 5 & 0.16 & 63.14 & 67.45 \\
\hline & 10 & 0.18 & 65.47 & 68.86 \\
\hline & 20 & 0.20 & 68.19 & 71.64 \\
\hline & 40 & 0.31 & 73.45 & 77.23 \\
\hline & 10 & 0.47 & 87.18 & 91.18 \\
\hline & 100 & 0.53 & 93.97 & 93.97 \\
\hline
\end{tabular}


что видно из данных таблицы. Также в таблице приведены рассчитанные значения вязкости для полимерных смесей $\left(\eta^{\text {pacч }}\right.$ и $\left.\eta^{\text {aд }}\right)$ при частоте осцилляции 1000 Гц.

В-третьих, характер зависимости комплексной вязкости от соотношения полимеров в смеси определялся значением частоты осцилляции (угловой скорости). Так, если при небольших частотах осцилляции $(f=0.01-10$ Гц, что соответствует угловой скорости $w=2 \pi f$ в диапазоне от 0.06 до $60 \mathrm{c}^{-1}$ ) вязкость расплава смеси полимеров в общем случае отклонялась в большую сторону от аддитивных значений (Рис. 1a), то при больших значениях угловой скорости (частоты осцилляции) ситуация изменялась. Как видно из представленных на Рис. $1 \mathrm{~b}$ данных, значения комплексной вязкости, определенные экспериментально при частоте осцилляции 100 Гц имеют значения меньшие, нежели аддитивные. Так же в меньшую сторону отклоняются и значения комплексной вязкости при частоте осцилляции 1000 Гц, рассчитанные по определенным из экспериментальных данных, по сравнению с аддитивными значениями (Табл. 1).

Объяснить наблюдаемые явления можно следующим образом. Известно, что расплавы полимеров представляют собой жидкости с определенной структурой, вследствие формирования агрегатов из макромолекул. Определяя вязкость расплава при малых частотах осцилляции, имеется возможность охарактеризовать систему макромолекул с исходной, неразрушенной структурой. Тот факт, что вязкость расплава смеси полимеров, определенная при малых частотах осцилляции, характеризуется повышенными значениями (поскольку имеет место отклонение в большую сторону от аддитивных значений) свидетельствует о том, что макромолекулы двух разных полимеров взаимодействуют друг с другом, например, за счет образования гетероагрегатов - ПП и второго полимера (ПВД-20 или ПНД-276) [20]. Учитывая отсутствие термодинамической совместимости между двумя разными

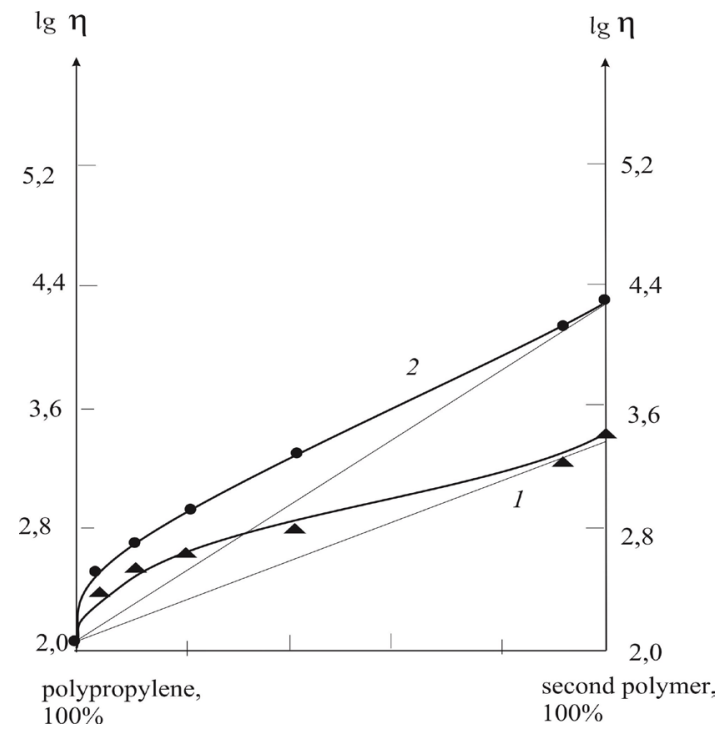

полимерами, такие гетероагрегаты ПП-второй полимер являются нестойкими и разрушаются при повышенных значениях скоростей сдвига. Данный факт носит, безусловно, положительный характер, поскольку пониженные значения вязкости смесевой композиции в существенной мере облегчают переработку.

Таким образом, композиции на основе смесей ПП-ПВД-20 и ПП-ПНД-276 будут (вследствие пониженных значений вязкости) достаточно легко перерабатываться при высоких скоростях сдвига, а повышенное взаимодействие полимеров между собой (при малых скоростях сдвига, или в их отсутствие) обеспечит им улучшенные деформационно-прочностные характеристики.

\section{4. Выводы}

1. Установлено, что увеличение содержания второго полимера в расплаве композиции на основе вторичного полипропилена приводит к закономерному увеличению вязкости и показателя $n$ в степенной зависимости вязкости от частоты осцилляции.

2. Экспериментальные реологические данные по значениям комплексной вязкости композиции на основе смесей двух полимеров (ПП с ПНД-276, ПВД-20), определенные при низких значениях частоты осцилляции, во всех случаях характеризуются повышенными (по сравнению с аддитивными) значениями, что говорит о взаимодействии компонентов смеси и формировании гетероагрегатов ПП-второй полимер.

3. Экспериментальные реологические данные по исследованию комплексной вязкости композиции на основе смесей двух полимеров, определенные при частоте осцилляции 100 Гц во всех случаях характеризуются пониженными (по сравнению с аддитивными) значениями, что свидетельствует о раз-

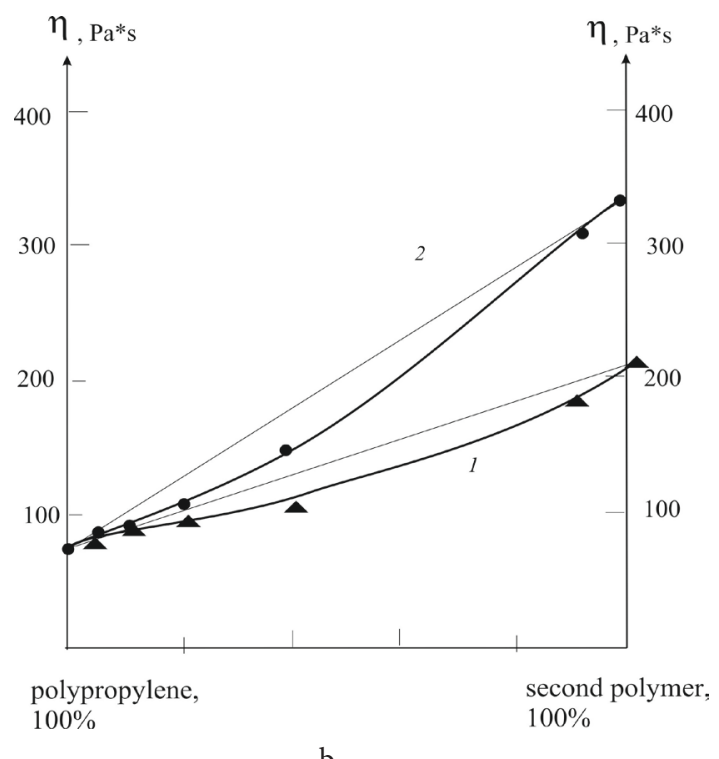

b

Рис. 1. Зависимость комплексной вязкости расплава смеси ПП с ПВД-20 (1) и ПНД-276 (2), определенная при частоте осцилляции 0.1 Гц (а) и 100 Гц (b), от соотношения полимеров в смеси.

Fig. 1. The dependence of complex viscosity of a melt of mix PP with HDPE-20 (1) and LDPE-276 (2) defined with a oscillation frequency of $0.1 \mathrm{~Hz}(\mathrm{a})$ and $100 \mathrm{~Hz}(\mathrm{~b})$ on a ratio of polymers in mix. 
рушении при больших скоростях сдвига формирующих ся гетероагрегатов ПП-второй полимер.

4. Значения комплексной вязкости, рассчитанные для значений скоростей сдвига, соответствующих условиям, характерным для переработки полимера методами экструзии и литья под давлением (до 10000 Гц), характеризуются пониженными (по сравнению с аддитивными) значениями, что позволяет предположить облегчение переработки смеси, по сравнению с переработкой индивидуальных полимеров.

Благодарности/Acknowledgements. Статья подготовлена в рамках выполнения научно-исследовательской работы в ФГБОУ ВО «Башкирский государственный университет» при финансовой поддержке Министерства образования и науки Российской Федерации (договор № 03.G25.31.0275). /This paper was prepared as part of the research work at the Bashkir State University, with financial support from the Ministry of Education and Science of the Russian Federation (contact № 03.G25.31.0275)

\section{Литература/Referencies}

1. G. Schramm. A Practical Approach to Rheology and Rheometry. 2nd ed. Gebrueder HAAKE GmbH, Karlsruhe. (2000) 291 p.

2. Yu. T. Panov, L. A. Chizhova, E. V. Ermolaeva. The modern methods of processing of polymeric materials. Extrusion. Injection molding: manual. Izd-vo VlGU, Vladimir, (2013) 128 p. (in Russian) [Ю.Т. Панов, Л. А. Чижова, Е.В. Ермолаева. Современные методы переработки полимерных материалов. Экструзия. Литье под давлением: учебное пособие. Изд-во ВлГУ, Владимир, (2013) 128 c.]

3. S. V. Vlasov, L.B. Kandyrin, V.N. Kuleznev et al. Bases of technology of processing of plastic: studies. for higher education institutions. Himiya, Moscow. (2004) 600 p. (in Russian) [С. В. Власов, Л.Б. Кандырин, B.Н. Кулезнев и др. Основы технологии переработки пластмасс: учеб. для вузов. Химия, Мосвка. (2004) 600 c.]

4. E.S. Cobkallo. Polyethylene, polypropylene and other polyolefins. Professiya, St. Petersburg. (2006) 256 p.

5. H. Makio, T. Fujita. 14th Asia Pacifil Confederation of Chemical Engineering Congress, Singapore. 760 (2012).

6. A.P. Belokurova, T. A. Ageeva. Himiya and technology of receiving polyolefins: manual. Ivan. state. chemical technol. un-t, Ivanovo. (2011) 126 p. (in Russian)
[А.П. Белокурова, Т.А. Агеева. Химия и технология получения полиолефинов: учебное пособие. Иван. гос. хим.-технол. ун-т, Иваново. (2011) 126 с.]

7. L. Guseva. Plastiks. 12, 84 (2009). (in Russian) [Л. Гусева. Пластикс. 12, 84 (2009).]

8. K. V. Goryachkina. Prioritetnyie nauchnyie napravleniya: ot teorii k praktike. 33, 169 (2016). (in Russian) [К.В. Горячкина. Приоритетные научные направления: от теории к практике. 33, 169 (2016).]

9. L. V. Chuprova, E.R. Mullina, O.A. Mishurina, O.V. Yershova. Sovremennyie problemyi nauki i obrazovaniya. 4, 212 (2014). (in Russian) [Л.В. Чупрова, Э.Р. Муллина, О.А. Мишурина, О.В. Ершова. Современные проблемы науки и образования. 4, 212 (2014).]

10. N.P. Prorokova, S. Yu. Vavilova, M.I. Biryukova, G. Yu. Yurkov, V.M. Buznik. Rossiiskie nanotehnologii. 9-10, 61 (2014). (in Russian) [Н.П. Пророкова, С.Ю. Вавилова, М.И. Бирюкова, Г.Ю. Юрков, В.M. Бузник. Российские нанотехнологии. 9-10, 61 (2014).]

11. A. E. Zaikin. Klei. Germetiki, Tehnologii. 8, 32 (2018). (in Russian) [А.Е. Заикин. Клеи. Герметики, Технологии. 8, 32 (2018).]

12. I. A. Kirsh, D.A. Pomogova, A.E. Chalyih, I.S. Tveritnikova. Plasticheskie massyi. 5-6, 5 (2018). (in Russian) [И. А. Кирш, Д.А. Помогова, А.Е. Чалых, И.С. Тверитникова. Пластические массы. $5-6,5$ (2018).]

13. H.G. Karian. Handbook of Polypropylene and Polypropylene Composites, Revised and Expanded. CRC Press, Boca Raton. (2003) 576 p.

14. H. P. Blom, J. W. The, T. Bremner, A. J. Rudin. Polymer. 39, 4011 (1998).

15. C.M. Tai, R. KY. Li, C.N. Ng. J. Polymer testing. 2, 143 (2000).

16. L.S. Shibryaeva, A.A. Popov, G.E. Zaikov. Thermal Oxidation of Polymer Blends. VSP, Leiden, Boston. (2006) $248 \mathrm{p}$.

17. S. Jose, A. S. Aprem, B. Francis et al. Eur. Polym. J. 9, 2105 (2004).

18. L. A. Utracki. Commercial polymer blends. Chapman and Hall, London. (1998) 658 p.

19. S. Doroudaini, C. B. Park, M. T. Kortschot. Polym Eng Sci. 7, 1205 (1998).

20. V.N. Kuleznev. Polymer blend. Chemistry, Moscow. (1980) 304 p. (in Russian) [В.Н. Кулезнев Смеси полимеров. Химия, Москва. (1980) 304 с.] 